\title{
Les Mémoires du baron Georges Cuvier (1833) de Mistress Lee : mémoires scientifiques, pacte biographique, ou réécriture des savoirs ?
}

En 1833 paraissent simultanément à Paris, à Londres et à New York les Mémoires $d u$ baron Georges Cuvier publiés par Mistress Lee en français et en anglais. ${ }^{1}$ La bonne réception de cet ouvrage des deux côtés de l'Atlantique et de la Manche est liée à la réputation à la fois étendue et problématique de ce grand homme scientifique après sa mort en 1832, aussi bien qu'à l'autorité réputée de sa mémorialiste maintenant oubliée, Sarah Bowdich/Mistress R. Lee (1791-1856). Quoique l'importance de Cuvier dans l'histoire et le développement des sciences au Muséum national d'histoire naturelle parisien soit éclipsée en 1870 par ses successeurs à la chaire d'anatomie comparée, les mérites de ces Mémoires (version française) étaient toujours reconnus en cette période d'échec national. Cet ouvrage témoigne de la disparition parmi tant d'autres de leur traducteur en français, l'entomologiste renommé Théodore Lacordaire (1801-1870). Le fait que Charles Morren ${ }^{2}$ le biographe de Lacordaire lui attribue également leur rédaction, en raison de son autorité scientifique, tient à son rôle ambigu dans la production du texte d'après

1 Les titres et détails bibliographiques exacts sont respectivement : Mémoires du baron Georges Cuvier, publiés en anglais par Mistress Lee, et en français par M. Théodore Lacordaire sur les documents fournis par sa famille : Théodore Lacordaire : Mémoires du baron Georges Cuvier. Paris : H. Fournier 1833 ; Mrs R. Lee (formerly Mrs. T. Ed. Bowdich) : Memoirs of Baron Cuvier. Londres : Longman, Rees, Orme, Brown, Green \& Longman 1833 et Mrs R. Lee (formerly Mrs. T. Ed. Bowdich) : Memoirs of Baron Cuvier. New York : J. \& J. Harper 1833. La version française sera abréviée $M B C f r$ pour les citations incluses ci-dessus. Je remercie ma collègue, Élodie Laügt, de ses observations et interventions portant sur les versions préparatoires de cet article.

2 Charles Jacques Edouard Morren : Éloge de Jean-Theodore Lacordaire. Liège : J. Desoer 1870. Il affirme que Lacordaire " paye un juste tribut à son illustre maître par la publication française en 1833 des Mémoires sur [sic] Georges Cuvier de Mistress Lee » (p. 11). Qu'il s'agisse en fait de sa traduction de ce texte, cela se voit avec plus de transparence dans les références bibliographiques (p. 25) qui terminent le chapitre en question. Aujourd'hui encore, il est à noter que l'article électronique consacré à Lacordaire, sur la version française du site Wikipédia, répète cette même erreur d'attribution, voir URL : https://fr.wikipedia.org/wiki/Th\%C3\% A9odore_Lacordaire [consulté le 13/02/2019].

Mary Orr, University of St Andrews

Ә Open Access. (C) 2020 Mary Orr, published by De Gruyter. (c) BY-NC-ND This work is licensed under a Creative Commons Attribution-NonCommercial-NoDerivatives 4.0 International License. 
la page de titre dans l'édition Fournier [Figure 1]. Lacordaire occupait en 1830, sous Cuvier, un poste au Muséum national d'histoire naturelle de Paris. Il avait ainsi assisté de très près aux débats amers de 1830 concernant la célèbre « Querelle des Analogues » entre Cuvier et Étienne Geoffroy Saint-Hilaire autour de la notion de fixité, ou de transformisme des espèces. ${ }^{3}$ La mort inattendue de Cuvier en 1832 tombe à la fin de cet échange amer et passionné, qui n'avait remporté aucune victoire pour lui, leur vainqueur supposé.

Ces faits divers et diversement bio(lo)graphiques soulignent l'importance $\mathrm{du} \mathrm{XIX}^{\mathrm{e}}$ siècle français comme le siècle de grandes révolutions politiques, sociales et scientifiques. ${ }^{4}$ Les débats importants concernant les formes de la vie biologique qu'incarnent la Querelle de 1830 et ses suites - comme par exemple le transformisme, l'adaptation, l'extinction, la tératologie, les origines humaines - signalent aussi un changement séismique de paradigmes scientifiques. La Vie avec une majuscule d'un biologiste éminent, ou d'un grand explorateur scientifique de cette période possède ainsi une valeur biographique supplémentaire, qui se distingue qualitativement d'une grande vie artistique par son objet. Le curriculum vitae scientifique exceptionnel, parce que voué à de nouvelles recherches de la biosphère, non seulement augmente les savoirs humains, mais les repense aussi plus radicalement et définitivement. Cependant, pour les détracteurs de Cuvier depuis 1832, c'est lui l'instigateur des classifications et théories intransigeantes et antirévolutionnaires - même s'il les a nommées « les révolutions du globe $»^{5}-\mathrm{du}$ fait qu'il avait mis en évidence plusieurs extinctions cataclysmiques expliquant les espèces sans semblables modernes. De la même manière dans ses fonctions politiques à l'Institut et au Muséum national d'histoire naturelle de Paris, Cuvier était considéré comme l'obstacle principal au progrès et à l'évolution naturelle de la science moderne (transformiste), ainsi qu'aux carrières de ses chercheurs.

La mort d'un génie scientifique signalerait ainsi un tournant majeur, un état potentiel d'échec et de rupture, susceptible de mettre en marche un changement de paradigmes. La signification de la disparition inattendue de Cuvier en 1832 se voit peut-être le plus visiblement dans l'absence surprenante d'Éloge

3 Pour une analyse approfondie de cette querelle, voir Toby A. Appel : The Cuvier-Geoffroy Debate: French Biology in the Decades before Darwin. Oxford : Oxford University Press 1987.

4 Voir à ce sujet Thomas S. Kuhn : La Structure des révolutions scientifiques. Traduit par Laure Meyer. Paris : Flammarion 1983.

5 Georges Cuvier : Discours sur les révolutions de la surface du globe et sur les changements qu'elles ont produits dans le règne animal (1822). Il a rédigé une Histoire des progrès des sciences naturelles depuis 1789 jusqu'à ce jour en 5 volumes de 1826 à 1836. 


\title{
MÉMOIRES
}

DU BARON

\section{GEORGES CUVIER,}

PCBI,lés EN ANGLAIS

PAR MISTRESB LEE,

ET EN TRANÇAIS

\section{PAR M. THÉODORE LACORDAIRE,}

SUR LES DOCUMENS FOURNIS PAR SA FAMILLE.

\author{
PARIS, \\ H. FOURNIER, LIB RAIRE, \\ RUE DF. SPINE, NO 14 . \\ M DCc:C $\times \times \times 111$.
}

Figure 1: La page de titre de l'édition Fournier des Mémoires du baron Georges Cuvier (1833). 
scientifique officiel en son honneur, ou prononcé lors de ses funérailles. ${ }^{6}$ Dans son rôle de Secrétaire perpétuel du Muséum national, Cuvier avait rédigé des rapports scientifiques annuels publics sur les découvertes et publications de ses collègues. Au moment du décès de l'un d'eux, il publiait l'Éloge scientifique qui rendait hommage à ce collègue et à ses contributions aux sciences naturelles. ${ }^{7}$ Son dernier Éloge publié avait célébré la vie scientifique de son antagoniste transformiste, Jean-Baptiste Lamarck (1744-1829). Cuvier, le maître de cette forme biographique scientifique, n'avait donc ni prédécesseur ni successeur, et ne reçut pas d'Éloge, quoiqu'il ait lui-même préparé d'avance le sien (qui ne fut pas utilisé). ${ }^{8}$ En l'absence d'un confrère mémorialiste parmi les chaires du Muséum, il fallut attendre 1834 pour que soit publié l'Éloge historique de Cuvier par son disciple, Jean Pierre Flourens (1794-1867). ${ }^{9}$ En imitant fidèlement le modèle et le titre officiel de son prédécesseur, son emploi de l'adjectif « historique » n'eut pour effet que de souligner davantage une forme générique en train de disparaître, voire dépassée, parce que privée de l'autorité scientifique que Cuvier lui avait conférée à son zénith.

L'absence de biographe officiel et de biographie nationale et internationale est pourtant comblée en 1833 par les Mémoires du baron Cuvier/Memoirs of Baron Cuvier de Mistress Lee. En France, cette publication à double portée ne fait que mettre en lumière l'embarras causé par Cuvier quant à la possibilité de le remplacer dans sa fonction de mémorialiste officiel du Muséum, mais aussi

\footnotetext{
6 À la séance du 17 décembre 1832 de la Chambre des Pairs son Président, M. le Baron (Étienne-Denis) Pasquier prononce l'Éloge de M. le Baron Georges Cuvier, mais il admet ouvertement son incapacité à rendre justice aux œuvres savantes de Cuvier. Pasquier n’y fait référence qu'en passant à un morceau choisi dans l'Histoire des Ossements fossiles. Ce texte est reproduit sur le site Gallica de la BNF : http://gallica.bnf.fr/ark:/12148/bpt6k90326b [consulté le 13/02/2019]. À Besançon, Charles Léopold Laurillard prononça un Discours sur Cuvier : Éloge de M. Le Bon Cuvier par C.-L. Laurillard. Discours couronné par l'Académie des Sciences, Belles Lettres et Arts de Besançon. Paris : Levrault 1833.

7 Georges Cuvier : Éloges historiques des membres de l'Académie royale des sciences, lus dans les séances de l'Institut royal de France, 3 vol. (1819-1827), disponibles dans une édition nouvelle de 1861 publiée par Firmin sur le site Gallica de la BNF : http://gallica.bnf.fr/ark:/12148/ bpt6k862478.r=.langEN [consulté le 13/02/2019]. Cuvier y a aussi reconnu des hommes scientifiques majeurs étrangers, comme Sir Humphry Davy.

8 Cuvier avait préparé une sorte de pré-éloge autobiographique pendant les années 1820, des " Mémoires pour servir à celui qui fera mon éloge », reproduits dans Jean Pierre Flourens : Recueil des Éloges historiques lus dans les séances publiques de l'Académie des Sciences. Paris : Garnier Frères 1856.

9 Jean Pierre Flourens : Analyse raisonnée des travaux de Georges Cuvier, précédée de son éloge historique. Paris : chez Paulin 1841.
} 
du fait de son statut de grand homme scientifique mondial et de cause célèbre nationale. Comment commémorer cette grande Vie scientifique sans parler de ses activités scientifiques, qui dans le cas de Cuvier dépassent les compétences d'amateur d'un biographe « historien des sciences »? Comment aussi rendre hommage à ses rôles nationaux officiels quand on n'est pas Français ? Abordant directement ces deux sujets capitaux qu'incarne l'Éloge scientifique à la Cuvier - dont deux longues parties élaborent avec une lucidité rare l'importance des œuvres de Cuvier, et de ses rôles scientifiques administratifs - cet ouvrage de Lee représente un choc profond que nous considérons ici pour la première fois. Sa plume d'auteure étrangère lance un grand défi ouvertement caché à l'ordre fixe de la science établie et de ses pratiques, étant donné l'impossibilité pour une femme de faire figure d'autorité scientifique à l'époque. ${ }^{10}$ Comment Mistress Lee arrive-t-elle alors à écrire ce qui est l'Éloge officiel de Cuvier sous d'autres termes ? Et si elle imite par cette réécriture les genres du modèle paradigmatique de la biographie scientifique nationale, réussit-elle aussi à le transformer?

\section{Le modèle paradigmatique de la Vie scientifique : mémoires et pacte biographique}

Pour l'histoire et la littérature des sciences du XIX ${ }^{\mathrm{e}}$ siècle français, les figures et les formes principales qui inspirent les mémoires d'une grande Vie savante et sa représentation, sont doubles. Cuvier exemplifie le premier modèle : ce génie et grand fondateur de systèmes scientifiques novateurs opère principalement dans son laboratoire. Si l'anatomie comparée et la paléontologie lui sont dues, leur développement dépend des collections croissantes du Muséum national, qui arrivaient des quatre coins du monde. Le grand explorateur scientifique international, qui les fournissait et les enrichissait par ses nouvelles découvertes, constitue l'autre grand modèle exemplaire qu'incarne Alexandre de Humboldt. C'est à lui que l'on doit un certain nombre de paradigmes scientifiques nouveaux - la géographie des plantes, l'écologie et les connaissances relatives à des courants climatiques intercontinentaux entre autres - phénomènes qu'il avait observés au cours de ses voyages dans les régions équinoxiales et russes

10 Voir à ce sujet Jean-Pierre Poirier : Histoire des femmes de science en France : du Moyen Âge à la Révolution. Paris : Pygmalion Gérard Watelet 2002 et Éric Sartori : Histoire des femmes scientifiques de l'antiquité au $\mathrm{XX}^{e}$ siècle : les filles d'Hypatie. Paris : Plon 2006. 
du globe. Ces deux hommes ont ainsi perfectionné les deux genres biographiques paradigmatiques de l'époque, présentant dans une sorte d'équivalence leur approche scientifique majeure et le mode empirique de communication de la vie savante. En ce qui concerne Cuvier, l'Éloge historique scientifique de ses pairs en France et à l'étranger rend aussi possible sa propre inscription à venir dans une " grande galerie » d'hommes scientifiques notables. Dans le cas de Humboldt, la rédaction de ses grands voyages scientifiques allait toujours de pair avec ses réflexions synthétiques géo-métaphysiques. Son Cosmos couronnera sa carrière vouée à la revendication de la Vie biogéographique, qui ne célèbre l'être humain qu'en tant qu'il participe à la pléthore de formes multiples qui répondent à leurs environnements particuliers. ${ }^{11}$

En créant des protagonistes et (anti-)héros savants fictifs, Balzac (Louis Lambert, 1835) Flaubert (La Tentation de saint Antoine, 1874) et Verne (Vingt mille lieues sous les mers, 1869) illustrent de manière diversement mémorable ces deux modèles paradigmatiques de l'homme scientifique au XIX ${ }^{\mathrm{e}}$ siècle. La narration des aventures et exploits presque surhumains de ces protagonistes constitue une odyssée moderne, qui explore les mondes nouveaux et souvent merveilleux de la découverte. Si Balzac et Flaubert ont peut-être envisagé dans leur œuvre fictionnelle respective l'impulsion folle ou monstrueuse du désir humain savant moderne, la volonté d'ajouter aux connaissances humaines et de tout apprendre/ comprendre reste le fond biographique des mémoires scientifiques et leur mesure de la réussite et du génie dans ces domaines. Il ne suffit pas d'observer avec fidélité son monde : il faut l'interroger et l'envisager autrement. Pourtant, ces écrivains fortement informés par les sciences naturelles positivistes de leur époque ne mettent pas en cause le statut culturel de leur personnage principal, qui dans son individualisme prométhéen et sa vocation savante ne se distingue pas véritablement des grands héros saints ou militaires. ${ }^{12}$ Dans l'histoire de la

11 Pour lui, chaque élément de la biosphère s’ajoute à la Vie organique locale et planétaire collective, grâce à des interactions environnementales multiples.

12 Voir Peter France : From Eulogy to Biography: the French Academic Éloge. In: Id./William St Clair (éds.) : Mapping Lives: the Uses of Biography. Oxford : Oxford University Press 2002, p. 83-101, où il note le modèle standard pour l'Éloge scientifique, à savoir « the heroic biography of a scientist, with its obligatory mention of the early signs of genius and the encounter with opposition » (p. 94). Dans le numéro spécial dirigé par David R. Oldroyd sur la « Biography » dans la revue Earth Sciences History 13, 1 (2013), les noms et les adjectifs choisis dans plusieurs titres exemplifient cet aspect hérö̈que - par exemple Wolf Mayer : William Noel Benson (1885-1957): Insights into the Life and Work of an Eminent Geologist ; Stephen Rowland : The Life and Geological Writing of the "Father of Russian science": Mikhail Lomonosov ; et Johannes Mattes : Alexander von Mörk et Poldo Fuhrich: the Conception of Heroes in Cave Exploration in the Early Twentieth Century (c'est moi qui souligne). 
biographie scientifique, ${ }^{13}$ des mémoires scientifiques traitent de la vie et des œuvres d'hommes savants exemplaires comme de monuments nationaux. Ce sont des "lieux de mémoire », pour emprunter le concept de Pierre Nora. ${ }^{14}$

La question du sexe et du genre sexué des mémoires scientifiques ne se pose donc pas au XIX ${ }^{\mathrm{e}}$ siècle, et ne s'est posée que récemment dans l'histoire des mémoires de l'époque, ${ }^{15}$ y compris son sous-genre scientifique. Il se voit que le masculin pluriel de "Mémoires » vante presque automatiquement une Vie majuscule scientifique à laquelle le mémorialiste a aussi visiblement participé soit en partageant les domaines professionnels de son sujet, soit en faisant partie du cercle de ses connaissances intimes. Or la question du genre entourant les mémoires se complique parce que le mémoire désignant des écrits juridiques et scientifiques se trouve toujours doublé par les genres de la mémoire, et de son expression écrite, historique ou imaginaire. Selon Anne Coudreuse, la femme mémorialiste crée des sortes de « [p]laidoyer où s’articulent de manière très complexe l'intime et le politique [. . .] dans la tourmente de l'Histoire, cherchant, sans oublier qu'elle est femme, à faire l'œuvre d'historienne ". ${ }^{16}$ À titre d'exemple on peut citer les mémoires (historiques) de Mme de Genlis (1746-1830), de Mme de Staël (1766-1817) et de la duchesse d'Abrantès (1784-1838), qui démontrent très clairement que cet acte de (se) figurer une vie (auto)biographique politique - que cette vie soit historique ou imaginaire - n'a pas nécessairement de sexe, et donc inclut déjà en théorie et en pratique les capacités et contributions de femmes. Si on en identifie une,

13 Voir par exemple Thomas Söderqvist : The History and Politics of Scientific Biography. Aldershot : Ashgate Publishing Ltd. 2007.

14 Pierre Nora : Les Lieux de mémoire. Paris : Gallimard 1982-1994.

15 Voir l'excellent recueil Les Mémoires, une question de genre ? dirigé par Anne Coudreuse, dans Itinéraires 1 (2011). Pour des études indispensables sur les mémoires de femmes au XIX siècle français, voir Henri Rossi : Mémoires aristocratiques féminins, 1789-1848. Paris : Champion 1998, et Damien Zanone : Écrire son temps. Les Mémoires en France de 1815 à 1848. Lyon : Presses universitaires de Lyon 2006. Il ne s'agit pourtant pas dans ces ouvrages de mémoires/ femmes mémorialistes scientifiques. La question des femmes scientifiques autobiographes est traitée dans le contexte des sciences britanniques par Alice Jenkins : Writing the Self and Writing Science: Mary Somerville as Autobiographer. In : Juliet John/Alice Jenkins (éds.) : Rethinking Victorian Culture. Basingstoke : Macmillan Press 2000, p. 162-178. Le phénomène d'une femme scientifique (et par conséquent sa biographie officielle) est supposé impossible dans la première moitié du XIX ${ }^{\mathrm{e}}$ siècle. Voir la note 12 ci-dessus, mais aussi l'étude de Martha Vicinus : "Tactful Organising and Executive Power": Biographies of Florence Nightingale for Girls. In : Michael Shortland/Richard Yeo (éds.) : Telling Lives in Science: Essays on Scientific Biography. Cambridge : Cambridge University Press 1996, p. 195-213.

16 Anne Coudreuse : Présentation. In : Itinéraires 1 (2011), p. 9-12, ici p. 11. 
pourtant, il existe une longue histoire et une tradition d'amnésie culturelle, où la femme savante oubliée-retrouvée devient l'exception ou l'anomalie faisant la preuve de l'inégalité du génie : un grand homme peut le posséder naturellement ; une femme exceptionnelle ne l'acquiert que rétrospectivement et par association à un parent éminent, surtout quand il s'agit des sciences. ${ }^{17}$

\section{Le cas des Mémoires du baron Georges Cuvier (1833) de Mistress Lee}

Dans le premier paragraphe de son introduction de sept pages, Mistress Lee thématise directement sa position singulière pour l'époque de (femme) mémorialiste scientifique « afin d'échapper au reproche de présomption que doit naturellement encourir une personne étrangère aux sciences, qui essaie d'exposer la vie d'un savant aussi illustre que M. Cuvier ${ }^{18}{ }^{18}$ S'ensuit une digression stratégique de trois pages, qui décrivent l'impossibilité de trouver après la mort de celui-ci un rapport qui soit fidèle aux faits de sa vie, ou qui lui rende suffisamment justice au niveau professionnel, surtout selon les souvenirs très personnels de Lee : « il n'y avait que moi en Angleterre qui, ayant été admise dans le sein de la famille de M. Cuvier, pouvait parler avec certitude de certains événemens ». ${ }^{19}$ L'impossibilité de garder le silence en dépit de son deuil provoque la détermination de Lee « à révéler à [ses] compatriotes le plus noble présent que la nature eût fait à M. Cuvier, [...] son cœur. Tel est le but principal de cette biographie. ${ }^{20}$ Ce projet se veut ainsi un plaidoyer où s'articulent de manière très complexe l'intime et le scientifique, pour reprendre la formule de Coudreuse ci-dessus, de façon à mieux éclairer les raisons non souhaitées de la part de Lee pour l'écrire, raisons qui expliquent aussi sa signature de femme mariée étrangère, ses positions (auto)biographiques

17 Cette argumentation formulée par Pnina G. Abir-Am et Dorinda Outram dans Uneasy Careers and Intimate Lives: Women in Science 1789-1979. New Brunswick/Londres : Rutgers University Press 1989, a inspiré la redécouverte de ces sœurs, filles et épouses collaboratrices auparavant absentes ou secondaires dans les biographies officielles d'un William Herschel (Caroline Herschel), d'un lord Byron (Ada Lovelace), ou d'un Antoine Lavoisier (Marie-Anne Lavoisier), par exemple.

18 Théodore Lacordaire : $M B C f r$, p. 1. C'est moi qui souligne cette expression grammaticale qui fait aussi référence à une femme étrangère réelle.

19 Ibid., p. 4.

20 Ibid. 
et son autorité scientifique. En se servant tout d'abord d'un « nous » universel qui devient dans la seconde moitié de sa préface un « nous » plus personnel, Lee introduit et cache tout à la fois la singularité de sa personne dans les milieux scientifiques et familiaux de Cuvier : c'est grâce à l'arrivée en 1819 à Paris de feu M. Bowdich (1791-1824) son premier mari, l'explorateur renommé d'Ashanti en Afrique de l'Ouest. Sarah Bowdich (Lee) l'a accompagné dans ses voyages scientifiques, y compris son séjour de quatre ans à Paris afin qu'ils préparent ensemble une première mission scientifique en Sierre Léone. ${ }^{21}$ Ainsi munie de toute la respectabilité (professionnelle) nécessaire pour aborder son sujet, et en pleine reconnaissance de l'aide des collègues de Cuvier - MM. le baron Pasquier et Laurillard, le docteur Duvernoy et le baron de $\mathrm{H}$ [Alexandre de Humboldt] ${ }^{22}$ - Mistress Lee termine son introduction sur le plan en quatre parties de ses Mémoires :

La première donnera les dates des évènemens [sic] les plus importans de la vie de M. Cuvier. La seconde contiendra l'examen de ses divers ouvrages comme savant ou philosophe. La troisième sera consacrée à sa carrière législative ; et la quatrième enfin se bornera principalement aux anecdotes propres à faire connaître son caractère. En suivant cette marche, je pourrai peut-être tomber dans quelques répétitions ; mais j'espère qu'on me le pardonnera, si l'on considère que chaque partie formera ainsi un tout complet par elle-même, ce qui rendra les recherches plus faciles. ${ }^{23}$

Dans sa méthode pour aborder et construire son Éloge selon cette « table de matières ", Lee se révèle être une chercheuse empirique sérieuse qui émule l'Éloge historique de Cuvier à son propos. Cependant son témoignage dans la dernière partie pour mieux « faire connaître son caractère » dépend aussi de son cercle intime. En empruntant le titre de Mémoires (que Cuvier a choisi pour son ouvrage autobiographique préparé avant sa mort), Lee révèle sa reconnaissance non moins profonde à son amie et collaboratrice scientifique dans ce projet, Sophie Duvaucel (1789-1867). Cette dernière était la belle-fille

21 La meilleure note biographique de Mistress Lee est celle de Donald de B. Beaver : Lee, Sarah (1791-1856). In : Oxford Dictionary of National Biography. Oxford : Oxford University Press 2004 [en ligne] ; URL : http://www.oxforddnb.com/view/article/16310 [consulté le 13/02/ 2019]. Pour une étude approfondie de la vie scientifique de Sarah après la mort de T. Edward Bowdich en 1824 en Gambie, et ses collaborations scientifiques avec Cuvier après 1825 voir Mary Orr : Women Peers in the Scientific Realm: Sarah Bowdich (Lee)'s Expert Collaborations with Cuvier, 1825-1833. In : Notes and Records 69, 1 (mars 2015), p. 37-52.

22 Ces noms se trouvent dans une seule note révélatrice des sources scientifiques françaises sans exception de Lee (Théodore Lacordaire : $M B C f r, p .7$ ), mais ils indiquent aussi ses rapports scientifiques personnels avec des confrères proches de Cuvier. Voir la note 6 pour les ouvrages en question du baron Pasquier et de Laurillard.

23 Théodore Lacordaire : MBCfr, p. 7-8. 
de Cuvier et comptait parmi ses femmes « aides naturalistes " non-officielles. ${ }^{24}$ C'est Sophie qui a fourni à Sarah Lee - et d'ailleurs au baron Pasquier aussi - certains documents personnels de son beau-père pour enrichir les informations de ces Mémoires scientifiques. Mais la correspondance précieuse d'un autre parent de Cuvier et parrain de Sophie, le docteur G.-L. Duvernoy, ${ }^{25}$ contient des lettres en français que ce dernier a reçues en février 1833 d'une « Mistress R. Lee », laquelle hésite longtemps avant d'accepter la commission de Mme Cuvier (secondée de Sophie Duvaucel), à savoir la rédaction de cet Éloge (officiel) de Cuvier. Lee n'en fait aucune mention dans l'introduction à ses Mémoires, pour éviter non seulement tout « reproche de présomption » mais aussi afin de garder en tout point la modestie de sa position singulière de femme mémorialiste et de femme scientifique. ${ }^{26}$ À cet égard, il convient de noter la position non moins difficile dans les années 1820 et 1830 pour les femmes littéraires en France, dont le cas le plus proche de Lee est celui de George Sand. Sans son choix de nom de plume masculin, elle aurait peut-être moins facilement obtenu en 1832 le succès de son premier roman Indiana, qui lance sa « carrière » de femme de lettres. ${ }^{27}$

Qu'une femme écrivain/biographe scientifique se trouve difficilement acceptée parmi ses critiques contemporain(e)s, aussi bien que rétrospectivement, alors même qu'elle réussit dans un monde dit masculin ou impossible à l'époque, se voit immédiatement dans l'embarras évident que causent les Mémoires du baron Georges Cuvier/Memoirs of Baron Cuvier parmi certains biographes récents de Cuvier, surtout ses apologistes modernes. Pour Philippe Taquet, ancien directeur du Jardin des Plantes, ${ }^{28}$ la version française des Mémoires de Lee ne mérite que quelques mentions en bas de page. Ces Mémoires sont pour lui surtout la source de quelques anecdotes attribuées à la vie scientifique débutante de Cuvier, et qui ressemblent pour Taquet à des fables. Lee ne figure pas dans l'œuvre

24 Voir Mary Orr : Keeping it in the Family: the Extraordinary Case of Cuvier's Daughters. In : Cynthia Burek/Betty Higgs (éds.) : The Role of Women in the History of Geology. Londres : Geological Society 2007, p. 277-286.

25 Voir la lettre de Sarah Lee au docteur G.-L. Duvernoy du 18 fév. 1833 (no. 1708), MS 2749. In : Dr G.-L. Duvernoy : Correspondance. Paris : MNHN Bibliothèque Centrale 1833, t. VII.

26 Pour une analyse des topoï de modestie chez des femmes écrivains, voir par exemple Lucie Desjardins : Entre sincérité et artifice. La mise en scène de soi dans le portrait mondain. In : Tangence 77 (2005), p. 143-155. DOI : 10.7202/011703ar [consulté le 13/02/2019].

27 C'est Sand qui remettra en question plus tard dans Consuelo (1842) et La Comtesse de Rudolstadt (1844) la situation sociale précaire de la femme de génie singulière qu'est l'artiste compositeur. Tout dépend de sa modestie et de son mode de vie bienséant pour qu'elle voile et dévoile le mieux ses dons supérieurs et superlatifs.

28 Philippe Taquet : Georges Cuvier. Paris : Odile Jacob 2006. 
majeure du grand géologue et apologiste anglais de Cuvier, Martin J. S. Rudwick, ${ }^{29}$ quoiqu'il ait dû en avoir connaissance. Son étudiante doctorale, l'historienne (féministe) des sciences Dorinda Outram, mine la version anglaise des Mémoires de Lee dans les notes copieuses à son histoire socio-scientifique de Cuvier ${ }^{30}$ développée de sa thèse. Pourtant, Outram ne commente pas la signification historique singulière au point de vue générique de cet ouvrage, y compris le sexe de son auteur. Le modèle et pacte biographiques inconscients pour ces historiens modernes restent en effet l'Éloge historique à la Cuvier, à savoir la forme classique inchangeable discutée ci-dessus de " grand homme-grande œuvre », et donc ne nécessitant pas de préface pour l'introduire. L'embarras que crée ce supplément de Lee justifiant et exposant son nom et son autorité de femme écrivain britannique met clairement en évidence les préjugés à la fois génériques et de genre enracinés, auxquels Lee fait face dans son introduction. Cela explique également le fait qu'elle établisse avec soin les mérites de sa plume audacieuse aussi littéraire que scientifique. L'attention non moins scientifique de la critique littéraire - par exemple celle portée aux sous-textes génériques, stylistiques et narratifs comme l'emploi des topoï de modestie - révèle pourtant comment et pourquoi les Mémoires de Lee représentent un cas d'étude qui met aussi en question la nécessité d'une rupture nette à même de marquer son effet radical dans un changement de paradigmes.

Le style soigné de cette introduction de Lee exemplifie de manière comparative tous les mérites stylistiques littéraires des femmes mémorialistes (historiques) de l'époque en France comme Mme de Genlis. Il va de soi que ce même style neutre et objectif de biographe scientifique, c'est-à-dire sans sexe déterminé, domine cet ouvrage de Lee, car elle se concentre explicitement sur des faits élargis et entrelacés dans les quatre parties indépendantes de son étude qui constitue et compose dans son ensemble une vie scientifique extraordinaire. L'effet et l'objectivité de son regard d'étrangère et d'amie personnelle présentent $\mathrm{Cu}$ vier de nouveau à ses lecteurs, mais comme si c'était Cuvier lui-même qui se construisait sous leurs yeux, et à la manière de ses expositions publiques célèbres d'anatomie comparée au Jardin des Plantes, où il recréait à partir d'une seule des dents la forme complexe de son propriétaire. Ce n'est qu'après avoir

29 Martin J. S. Rudwick : Georges Cuvier, Fossil Bones and Geological Catastrophies: New Translations and Interpretations of Primary Texts. Chicago : Chicago University Press 1997.

30 Dorinda Outram : Georges Cuvier: Vocation, Science and Authority in Post-Revolutionary France. Manchester : Manchester University Press 1984. Cette étude reprend deux de ses publications précédentes, à savoir Dorinda Outram : Scientific Biography and the Case of Georges Cuvier: with a Critical Bibliography. In : History of Science 14, 2 (1976), p. 101-137 et Dorinda Outram : The Language of Natural Power: the « Éloges » of Georges Cuvier and the Public Language of Nineteenth Century Science. In : History of Science 16, 3 (1978), p. 153-178. 
exposé le portrait scientifique en grand format de Cuvier résultant de sa vie et l'importance de ses œuvres que Lee ose introduire dans la dernière partie de son ouvrage des mémoires plus personnel(le)s. S'il s'agit d' " anecdotes », ou d'épisodes captant la vie quotidienne privée de Cuvier, dont Lee était le témoin pendant ses séjours parisiens où elle était logée chez lui, elle les raconte avec la même autorité raisonnée qui soutient les parties précédentes. De cette manière elle anticipe la méthode scientifique paradigmatique de la biographie humaine, l'anthropologue pratiquant l'observation participante.

Sur le plan formel, Lee offre à ses lecteurs avertis en France et dans le monde anglophone une version de l'Éloge scientifique qui continue sans aucune différence visible le modèle officiel historique de Cuvier. Les deux premiers panneaux des Mémoires du baron Georges Cuvier nous présentent sa vie et sa formation scientifique selon les paramètres chronologiques classiques de la biographie scientifique, c'est-à-dire fortement documentée par les témoignages de ses collègues scientifiques du Jardin des Plantes de Paris. La toile de fond de ce contexte intellectuel permet à Lee, dans le deuxième volet, le plus long de son ouvrage, non seulement de décrire les œuvres séminales majeures que Cuvier y a produites ${ }^{31}$ comme elle le prétend, mais aussi de les commenter et de les juger par la preuve de leurs contributions nouvelles aux savoirs nationaux et internationaux. Dans un tour de force autoréflexif - une mise en abyme littéraire avant la lettre - Lee termine son résumé critique en discutant longuement les Éloges de Cuvier (y compris son dernier volume posthume) pour conclure sur le cursus de ses cours magistraux, interrompus par la tragédie de la mort de sa fille (Clémentine) en 1827. La reproduction d'une note personnelle (de son collègue Achille Valenciennes) à ce sujet pour terminer cette troisième partie capte sans besoin d'explication le but principal de sa biographie déjà signalé dans l'introduction, les qualités inébranlables de «son cœur».

L'effet littéraire de ces tours de force génériques signale ainsi sa plume de femme écrivain scientifique adroite, s’insérant avec une fidélité suprême dans la tradition et la composition des Éloges et des mémoires scientifiques officiels et historiques de leur intérieur. En même temps, le paradoxe de sa position d'observatrice étrangère ouvre maintenant les Mémoires de Lee à une transformation plus radicale du genre, et ce par rapport à la personne même qui l'avait établi. Elle ajoute dans une troisième partie une analyse non moins fidèle des

31 Voir l'incipit de cette deuxième partie : Théodore Lacordaire : $M B C f r$, p. 50 : «J'arrive à la partie [...] où mon sujet acquiert une grandeur et une importance que la vie seule de M. Cuvier pouvait fournir et, quoique je me sois bornée à une simple esquisse de ses travaux scientifiques, elle surpassera les autres parties en étendue. » 
devoirs publics nationaux de Cuvier, et de son dévouement aux bénéfices de la science et à sa dissémination : des instituts nationaux au niveau supérieur à l'enseignement national en France des sciences naturelles au niveau secondaire. ${ }^{32}$ En élargissant ainsi l'étendue scientifique et culturelle des rôles officiels de Cuvier, Lee renforce cet aspect de sa vie et de son œuvre - son âme publique et ses efforts législatifs pour améliorer les sciences - qui dépassent tout intérêt politique (scientifique, national) plus partisan. Cette troisième partie sert ainsi d'affiliation générique nouvelle renouant la forme officielle de l'Éloge historique à son élargissement futur civique, pour mieux démontrer la carrière scientifique (vie et œuvres) monumentale de Cuvier comme pierre de touche pour ses successeurs.

L'ajout d'un quatrième et dernier volet opère cependant un dévoilement et une transformation plus définitive vis-à-vis du statut des précédents en délimitant les paramètres modèles de l'Éloge historique officiel. Tout en replaçant Cuvier au sein de sa famille et de sa vie quotidienne scientifique, ce supplément final ne témoigne de la vie intime, affective et sociale de Cuvier - entre amis de son salon du samedi par exemple - qu'en raison de la coprésence (auto)biographique singulière de $s a$ biographe. Ce volet, le plus court, fait ainsi exploser définitivement les mythes - ou les «fables » pour revenir à ce terme péjoratif de Taquet pour désigner des aspects anecdotiques merveilleux de faux mémoires d'une grande réputation scientifique (ou artistique), qui fait semblant de se construire indépendamment de tout support : conjugal-familial, professionnel, amical, et de l'étranger.

C’est en appliquant avec la plus grande fidélité la forme générique officielle de l'Éloge à la Cuvier dans ses Mémoires à Cuvier lui-même que Sarah Lee réussit un Éloge suprêmement personnel, qui se distingue en même temps de sa forme paradigmatique grâce à un remodelage plus hybride et pluriel, souligné par les ajouts qu'elle y fait : sa signature, son introduction et les deux dernières parties supplémentaires. De cette manière, elle s'insère dans la tradition de l'Éloge officiel (français), mais s'en distancie en tant qu'il est la seule forme (institutionnelle) de la biographie scientifique nationale. En se révélant finalement dans les cercles professionnels, et intimes, de Cuvier cette « personne étrangère » et grande mémorialiste brise le pacte biographique des Mémoires scientifiques nationaux de manière formelle, générique, et aussi autobiographique.

32 Elle n'oublie pas les rôles que Cuvier avait joués en tête du Rétablissement des religions non catholiques de France en 1808. 


\section{Les Mémoires et après}

La métamorphose du mémoire et la transformation des mémoires que représentent les Mémoires du baron Georges Cuvier ne comblent pourtant pas le grand vide personnel de Lee en 1833, quoiqu'elle lui rende hommage comme à son mentor scientifique. De 1826 à 1832 Sarah (Bowdich, ensuite Mistress Lee) a compté parmi les correspondants étrangers de Cuvier les plus importants pour son ichtyologie définitive, l'Histoire naturelle des poissons (1828-1848), qu'il prépare avec Achille Valenciennes. Elle a demeuré chez les Cuvier lors de ses séjours, dont l'objet premier était de lui fournir des dessins et des renseignements ichtyologiques spécialistes, puisés dans les ressources de la bibliothèque privée de Sir Joseph Banks à Londres. L'objet second de ces visites était ses propres recherches scientifiques dans les bibliothèques de Cuvier et dans les collections du Jardin des Plantes pour préparer une nouvelle histoire naturelle, qu'elle illustre elle-même en couleurs, The Fresh-Water Fishes of Great Britain [Les poissons d'eau douce de la Grande Bretagne], et qui sort dans des numéros réguliers entre 1828 et $1838 .^{33}$ Pour subventionner ces recherches et visites scientifiques, Sarah a contribué régulièrement à des revues culturelles et scientifiques britanniques nouvelles, comme le Magazine of Natural History, par ses articles savants - sous forme de mémoires sur l'état présent en France des sciences naturelles. En même temps et à l'invitation des éditeurs de nouveaux Gift Books de luxe à offrir aux jeunes femmes grand-bourgeoises, ${ }^{34}$ Lee a écrit des récits africains imaginaires fortement colorés par ses propres expériences " ethnographiques " pendant ses voyages d'exploration scientifique avec son premier mari en Ashanti (1818) et en Gambie (1824).

La mort de Cuvier en 1832 marque la fin définitive de ses visites et poursuites scientifiques à Paris et de ses contributions directes aux sciences naturelles en Grande-Bretagne. Comment continuer maintenant sa propre vie d'écrivain des sciences, qui pratique si bien ces formes génériques ? Il faut rappeler les deux grands modèles scientifiques et biographiques en France à

33 Voir mon article à son sujet, Mary Orr : Fish with a Different Angle: "The Fresh-Water Fishes of Great Britain” by Mrs Sarah Bowdich (1791-1856). In : Annals of Science 7, 1.2 (2014), p. 206-240.

34 Le « Gift Book » est un nouveau genre commercial britannique dans les années 1820. Une sorte de " keepsake " plus cultivé, il s'agit d'un livre-album-florilège illustré de gravures et destiné à être offert comme cadeau en guise de souvenir. Il a comme précédent en France les almanachs poétiques. Voir Catriona Seth : Les Muses de l'Almanach. La poésie au féminin dans l'Almanach des muses, 1789-1819. In : Christine Planté (éd.) : Masculin/Féminin dans la poésie et les poétiques du XIX siècle. Lyon : Presses Universitaires de Lyon 2002, p. 105-119. 
l'époque, Cuvier et Alexandre de Humboldt, pour mieux comprendre la solution bio(lo)graphique littéraire scientifique qu'elle trouve, et qui soutiendra sa carrière jusqu'à sa mort en 1856. Elle se relance et se recycle en 1835 en publiant un recueil de ses notes de voyage et de ses récits africains " ethnographiques » sous le titre Stories of Strange Lands [Histoires de pays étranges]. ${ }^{35}$ Cette fois-ci, elle souligne leur forme scientifique à la Humboldt plutôt qu'imaginaire : de longues notes scientifiques informées partout par sa vision " écologiste " décrivent les découvertes de mondes locaux africains d'un œil explorateur à la fois personnel et empirique. À ce tournant de sa vie maintenant délimitée par son exclusion du monde scientifique de la Grande-Bretagne du fait de son sexe, Sarah se tourne alors vers l'aspect formatif et éducatif des genres « scientifiques » et en dehors des voies officielles pour publier un mémoire scientifique. Ses fictions de sciences naturelles, des Aventures scientifiques pour adolescents, situées en Afrique de l'Ouest et en Australie $^{36}$ ont pour modèle l'explorateur scientifique Humboldt, ou encore son premier mari T. Edward Bowdich, mais en filigrane Sarah Lee s'y fait reconnaître pour elle-même. On ne peut pas si facilement classer ses descriptions et connaissances scientifiques sous les rubriques de la science dite « officielle » et « vulgarisée ». Par exemple, les animaux que Lee décrit dans leur environnement naturel, comme l'ornithorynque dans son Adventures in Australia (1851), sont ceux qu'elle a vus et dessinés dans les collections du Jardin des Plantes à Paris. Elle les explique à ses jeunes lecteurs et lectrices selon la science la plus récente relative à leur biologie (selon Cuvier) et à la nature de leurs habitats particuliers (selon Humboldt). En reprenant et en adaptant les formes encyclopédiques des savoirs, Lee réinvente ainsi dans sa réécriture des sciences naturelles pratiquées en France la découverte des aventures (mémoires) historiques, biographiques et autobiographiques qui représentent aussi une révolution silencieuse, profonde, et séismique en leur cœur. Étant donné que la science n'a pas de sexe selon Cuvier et Humboldt, cette révolution qu'effectuent des « personnes étrangères » dans les milieux

35 Mrs R. Lee : Stories of Strange Lands and Fragments from the Notes of a Traveller. Londres : Edward Moxon 1835.

36 Voir Mrs R. Lee : The African Wanderers or the Adventures of Carlos and Antonio. Embracing Interesting Descriptions of the Manners and Customs of the Western Tribes and the Natural Productions of the Country. Londres : Grant \& Griffith 1847 ; Adventures in Australia; or the Wanderings of Captain Spencer in the Bush and the Wilds. Containing Accurate Descriptions of the Habits and Natural Productions and Features of the Country. Londres : Grant \& Griffith 1851 ; Sir Thomas, or the Adventures of a Cornish Baronet in North-West Africa. Londres : Grant \& Griffith 1856. Pour une étude des fictions africaines de Lee, voir Silke Strickrodt : "Those Wild Scenes": Africa in the Travel Writings of Sarah Lee (1791-1856). Glienicke/Berlin et Cambridge/ Mass. : Galda \& Wilch 1998. 
officiels en France à partir de leurs laboratoires, voyages et publications scientifiques ne peut plus ignorer les contributions spécifiques et multiples de femmes.

\section{Conclusions}

Le cas des Mémoires du baron Cuvier de Lee nous a exposé dans ses divers aspects à un modèle officiel, et à sa transformation révolutionnaire radicale, à la fois inattendue et sans ruptures. C'est en opérant plutôt par ajouts majeurs avisés, et dans le but de faire revivre et d'élargir la forme de la biographie scientifique nationale que cette réécriture générique experte de Lee conserve en tout point la moelle de ses modèles paradigmatiques. En envisageant ses Mémoires comme la traduction, ou l'adaptation, littérale et figurative du pacte biographique scientifique pour mieux y révéler la communauté hétérogène qui l'informe - surtout la place des femmes, des étrangers et des " vulgarisateurs » parmi les « aides » naturalistes réels ou métaphoriques de Cuvier et de ses collègues - Lee met en lumière la face cachée vitale de toute réécriture de savoirs officiels et de biographies nationales de scientifiques, et ainsi les modèles qui renforcent leurs paradigmes. Il n'y a pas de portrait de Lee, qui l'aurait d'ailleurs démasquée en emportant ses topoï de modestie. En revanche, les productions de sa plume dans leur diversité surprenante de genres scientifiques et littéraires biographiques ne nous laissent pas oublier sa présence multiforme dès sa signature, qu'elle est femme écrivain, qu'elle fait œuvre d'historienne, de biographe, et de (femme) scientifique tout simplement parce que ces positions et disciplines n'ont pas de sexe (pré)déterminé. Ses Mémoires bilingues présentés ici pour la première fois dans des contextes de l'histoire de la biographie scientifique française du $\operatorname{XIX}^{\mathrm{e}}$ siècle invitent ses lecteurs modernes à contempler les traces et touches révélatrices d'autres femmes francophones et étrangères cachées au centre de l'histoire bio-(lo)graphique française et transnationale de cette époque avant tout révolutionnaire et transformiste.

\section{Bibliographie}

Abir-Am, Pnina G./Outram, Dorinda : Uneasy Careers and Intimate Lives: Women in Science 1789-1979. New Brunswick/Londres : Rutgers University Press 1989.

Appel, Toby A. : The Cuvier-Geoffroy Debate: French Biology in the Decades before Darwin. Oxford : Oxford University Press 1987. 
Beaver, Donald de B. : Lee, Sarah (1791-1856). In : Oxford Dictionary of National Biography. Oxford : Oxford University Press 2004 [en ligne] ; URL : http://www.oxforddnb.com/view/ article/16310 [consulté le 13/02/2019].

Coudreuse, Anne (éd.) : Les Mémoires, une question de genre ? In : Itinéraires 1 (2011).

Cuvier, Georges : Discours sur les révolutions de la surface du globe et sur les changements qu'elles ont produits dans le règne animal. Paris : Baudouin Frères 1826-1836.

Cuvier, Georges : Histoire des progrès des sciences naturelles depuis 1789 jusqu'à ce jour. 2 vol. Bruxelles : Société Belge de Librairies 1837-1838.

Cuvier, Georges : Recueil des éloges historiques lus dans les séances publiques de l'Institut royal de France. Nouvelle édition précédée de l'éloge historique de Georges Cuvier par Flourens. Paris : Librairie de Firmin Didot Frères, Fils \& $C^{\text {ie }} 1861$. URL : http://gallica.bnf. fr/ark:/12148/bpt6k862478.r=.langEN [consulté le 13/02/2019].

Desjardins, Lucie : Entre sincérité et artifice. La mise en scène de soi dans le portrait mondain. In : Tangence 77 (2005) p. 143-155. DOI : 10.7202/011703ar [consulté le 13/02/ 2019].

Duvernoy, Dr G.-L. : Correspondance. Paris : MNHN Bibliothèque Centrale 1833, t VII.

Flourens, Jean Pierre : Analyse raisonnée des travaux de Georges Cuvier, précédée de son éloge historique. Paris : chez Paulin 1841.

Flourens, Jean Pierre : Recueil des Éloges historiques lus dans les séances publiques de l'Académie des Sciences. Paris : Garnier Frères 1856.

France, Peter : From Eulogy to Biography: the French Academic Éloge. In: Id./William St Clair (éds.) : Mapping Lives: the Uses of Biography. Oxford : Oxford University Press 2002, p. 83-101.

Jenkins, Alice : Writing the Self and Writing Science: Mary Somerville as Autobiographer. In : Juliet John/Alice Jenkins (éds.) : Rethinking Victorian Culture. Basingstoke : Macmillan Press 2000, p. 162-178.

Kuhn, Thomas S. : La Structure des révolutions scientifiques. Traduction de Laure Meyer. Paris : Flammarion 1983.

Lacordaire, Théodore : Mémoires du baron Georges Cuvier. Paris : H. Fournier 1833. [MBCfr]

Laurillard, Charles Léopold : Éloge de M. Le Bon Cuvier par C.-L. Laurillard. Discours couronné par l'Académie des Sciences, Belles Lettres et Arts de Besançon. Paris : Levrault 1833.

Lee, Mrs R. (formerly Mrs. T. Ed. Bowdich) : Memoirs of Baron Cuvier. Londres : Longman, Rees, Orme, Brown, Green \& Longman 1833.

Lee, Mrs R. (formerly Mrs. T. Ed. Bowdich) : Memoirs of Baron Cuvier. New York : J. \& J. Harper 1833.

Lee, Mrs R. : Stories of Strange Lands and Fragments from the Notes of a Traveller. Londres : Edward Moxon 1835.

Lee, Mrs R. : The African Wanderers or the Adventures of Carlos and Antonio. Embracing Interesting Descriptions of the Manners and Customs of the Western Tribes and the Natural Productions of the Country. Londres : Grant \& Griffith 1847.

Lee, Mrs R. : Adventures in Australia; or the Wanderings of Captain Spencer in the Bush and the Wilds. Containing Accurate Descriptions of the Habits and Natural Productions and Features of the Country. Londres : Grant \& Griffith 1851.

Lee, Mrs R. : Sir Thomas, or the Adventures of a Cornish Baronet in North-West Africa. Londres : Grant \& Griffith 1856.

Morren, Charles Jacques Édouard : Éloge de Jean-Theodore Lacordaire. Liège : J. Desoer 1870. Nora, Pierre : Les Lieux de mémoire. Paris : Gallimard 1982-1994. 
Oldroyd, David R. (éd.) : Biography. In : Earth Sciences History 13, 1 (2013).

Orr, Mary : Keeping it in the Family: the Extraordinary Case of Cuvier's Daughters. In : Cynthia Burek/Betty Higgs (éds.) : The Role of Women in the History of Geology. Londres : Geological Society 2007, p. 277-286.

Orr, Mary : Fish with a Different Angle: "The Fresh-Water Fishes of Great Britain” by Mrs Sarah Bowdich (1791-1856). In : Annals of Science 7, 1.2 (2014), p. 206-240.

Orr, Mary : Women Peers in the Scientific Realm: Sarah Bowdich (Lee)'s Expert Collaborations with Cuvier, 1825-1833. In : Notes and Records 69, 1 (mars 2015), p. 37-52.

Outram, Dorinda : Scientific Biography and the Case of Georges Cuvier: with a Critical Bibliography. In : History of Science 14, 2 (1976), p. 101-137.

Outram, Dorinda : The Language of Natural Power: the «Éloges » of Georges Cuvier and the Public Language of Nineteenth Century Science. In : History of Science 16, 3 (1978), p. 153-178.

Outram, Dorinda : Georges Cuvier: Vocation, Science and Authority in Post-Revolutionary France. Manchester : Manchester University Press 1984.

Pasquier, Étienne-Denis : Éloge de M. le Baron Georges Cuvier. URL : http://gallica.bnf.fr/ ark:/12148/bpt6k90326b [consulté le 13/02/2019].

Poirier, Jean-Pierre : Histoire des femmes de science en France : du Moyen Âge à la Révolution. Paris : Pygmalion Gérard Watelet 2002.

Rossi, Henri : Mémoires aristocratiques féminins, 1789-1848. Paris : Champion 1998.

Rudwick, Martin J. S. : Georges Cuvier, Fossil Bones and Geological Catastrophies: New Translations and Interpretations of Primary Texts. Chicago : Chicago University Press 1997.

Sartori, Éric : Histoire des femmes scientifiques de l'antiquité au $x x^{e}$ siècle : les filles d'Hypatie. Paris : Plon 2006.

Seth, Catriona : Les Muses de l'Almanach. La poésie au féminin dans l'Almanach des muses, 1789-1819. In : Christine Planté (éd.) : Masculin/Féminin dans la poésie et les poétiques $d u x I x^{e}$ siècle. Lyon : Presses Universitaires de Lyon 2002, p. 105-119.

Söderqvist, Thomas : The History and Politics of Scientific Biography. Aldershot : Ashgate Publishing Ltd. 2007.

Strickrodt, Silke : "Those Wild Scenes": Africa in the Travel Writings of Sarah Lee (1791-1856). Glienicke/Berlin et Cambridge/Mass. : Galda \& Wilch 1998.

Taquet, Philippe : Georges Cuvier. Paris : Odile Jacob 2006.

Vicinus, Martha : "Tactful Organising and Executive Power": Biographies of Florence Nightingale for Girls. In : Michael Shortland/Richard Yeo (éds.) : Telling Lives in Science: Essays on Scientific Biography. Cambridge : Cambridge University Press 1996, p. 195-213.

Zanone, Damien : Écrire son temps. Les Mémoires en France de 1815 à 1848. Lyon : Presses universitaires de Lyon 2006. 